\title{
27 Beobachtungen von Vesico-Cervico-Uterinfisteln aus der Harnfistelcasuistik der Warschauer gyniatrischen Universitätsklinik des Docenten Dr. med. Ludwig Adolf Neugebauer (Vater).
}

(Fortsetzung der Arbeit: „Casuistik von 140 Vesico-Uterinfisteln66, s. diẹses Archiv, Band XXXIII, S. 270.)

Mitgetheilt von

Dr. med. Franz Ludwig Neugebauer (Sohn des Obigen), Ordin. der gyniatr. Univers.-Klinik des Spitals ,zum Heiligen Geist" in Warschau.

(Mit 17 Holzschnitten.)

Zu den im ersten Theile dieser Arbeit angekündigten 140 Fällen habe ich heute noch neun weitere Fälle hinzuzufügen, so dass die Gesammtanzahl der Fälle an Vesico-Uterinfisteln 149 beträgt. Ein Fall betrifft eine Beobachtung Dr. Rosenthal's aus jüngster Zeit, ein Fall ist neben dem schon früher sub $\mathrm{Nr}$. 56 unserer Gesammtcasuistik erwähnten Falle von Pippingsköld 1885 veröffentlicht (siehe im dritten Heft) und sieben gehören noch zu der Casuistik der gyniatrischen Klinik meines Vaters (1 Fall noch gegenwärtig in Behandlung).

1. (Fall 43 der Gesammtcasuistik): Fistula vesico-uterina. Sanatio spontanea. - Marie Haras, $28 \mathrm{j}$. VI para von mittlerer Körpergrösse, trat am 30. October 1862 wegen Harnincontinenz ein. Die erste und zweite Entbindung von je fünftägiger Dauer ergaben todtgeborene Kinder; das dritte Kind klein, lebend geboren. Vierte Schwangerschaft: Abort; fünfte Entbindung: natürliche Frühgeburt, Extraction einer siebenmonatlichen Frucht. Letzte Entbindung rechtzeitig. Am 18. Mai 1862 früh fünf Uhr Wehenbeginn, am 22. Mai sechs Uhr früh Wasserabfluss. 27 Stunden später natürliche Geburt eines todten Kindes. Manuelle Placentarlösung. Vom dritten Tage nach der Entbindung an Harnfluss durch die Scheide. Fünf Wochen bettlägerig. Incontinenz anfangs vollständig, später nur noch bei liegender Stellung, bei aufrechter jedoch (Gehen, Stehen, Sitzen) trocken. Die Kranke erschrak über die Wahrnehmung, dass ihre bald wieder eingetretene Periode durch die Harnröhre abfloss. - Befund: Retroversio-flexio uteri fixata, Perimetritis, Parametritis. Continenz im Stehen, sobald aber das Speculum in die Scheide eingeführt war, floss der Harn durch den Muttermund ab. Die Sonde gelangt 
146 Nengebauer, 27 Beobachtungen von Vesico-Cervico-Uterinfisteln.

vom Cervicalkanale aus durch eine $2 \frac{1}{2} \mathrm{~cm}$ oberhalb des Randes der vorderen Muttermundslippe gelegene Fistel in die Farnblase, wo sie mit dem durch die Harnröhre eingeführten Katheter zusammenstösst. Die Kranke verweigerte die ihx vorgeschlagene Hysterokleisis und wurde entlassen. Laut Bericht trat später Selbstheilung ein. (L. A. Neugebauer; L.-V. Nr. 50 a.)

2. (Fall 44 der Gesammtcasuistik): Fistula vesico-uterina. Heilung durch unmittelbaren Fistelverschluss mit einmaliger Operation (Syringokleisis cruenta immediata). - Rosalie Krupa, 29j. Bauersfrau aus Solec, IV para von mit. lerer Grösse. Erste und vierte (letzte) Entbindung bei Querlage mit Armvorfall beendigt von einer Babka ${ }^{1}$ ) mit Extraction je eines todten Kindes, zweite und dritte Entbindung regelmässig. Letzte Entbindung am 12. Juli 1870. Sofort Hamfluss durch die Scheide. Am 23. August 1870 trat die Kranke in das Spital ein und bekam alsbald ihre Periode wieder. -- Linksseitige Cervixfistel $13 \mathrm{~mm}$ oberhalb des Muttermundes. Kleine $1 \mathrm{~mm}$ weite Spalte. Nach einer erfolglosen Aetzung mit Höllenstein wurde nach vorausgeschickter Spaltung der vorderen Muttermundslippe bis in die Fistel hinein — so dass die Wunde ^förmig aussah - der unmittelbare Nahtverschluss ausgeführt. Nach Entfernung der Näte am achten Tage erwies sich die Wunde durch erste Vereinigung geheilt. Am 28. December 1870 geheilt entlassen. (L. A. Neugebauer; L.V. Nr. 50 b.)

3. (Fall 52 der Gesammtcasuistik): Fistula vesico-uterina. Heilung durch blutigen Verschluss des Muttermudes (Hysterokleisis cruenta). - Mereda Awrach (Nr. 104 des klinischen Fisteljournals), 25 jăhrige jüdische Schneidersfrau, I para, hinkte schon als Kind mit dem linken Beine. Trat am 8. October 1872 wegen Incontinenz ein. Die einzige Entbindung (vor sechs Jahren) war nach dreitägiger Dauer durch natürliche Austreibung eines todten Mädchens beendigt. Harnfuss durch die Scheide vom achten Tage an. Nach einiger Zeit besserte sich der Zustand soweit, dass die Kranke den Harn theilweise auf regelmässigem Wege zu entleeren lernte. Bei Besichtigung der Scheide suchte man vergebens nach einer Fistelöffnung und erst eine Milcheinspritzung in die Blase klärte den Sachverhalt auf. $13 \mathrm{~mm}$ oberhalb des Muttermundssaumes (s. Fig. 1) eine feine, nur $2 \mathrm{~mm}$ breite Cervicalfistel, in der Mittellinie gelegen. Periode regelmässig. Am 9. October 1872 Operation: Schnitt durch die vordere Lippe bis in die Fistelöffnung hinein; Anfrischung so, dass die Wunde sich ^förmig darstellte. Sechs Nahthefte, Verweilkatheter, letaterer musste jedoch, weil er Schmerzen verursachte, bald entfemt werden. Katheterisirt in Intervallen. Solange die Kranke lag, war sie trocken, - sobald sie jedoch aufstand, incontinent. Misserfolg, ebenso blieb ein zweiter und dritter

1) Babka ist die polnische Bezeichnung für eine niedere Stufe geburtshülflichen Hülfspersonales; die Babka erfüllt besonders auf dem Lande dort, wo noch keine gepriufte Hebamme ansässig ist, den Dienst der letzteren. 
Neug e bauer, 27 Beobachtungen von Vesico-Cervico-Uterinfisteln. 147

Fig. 1.

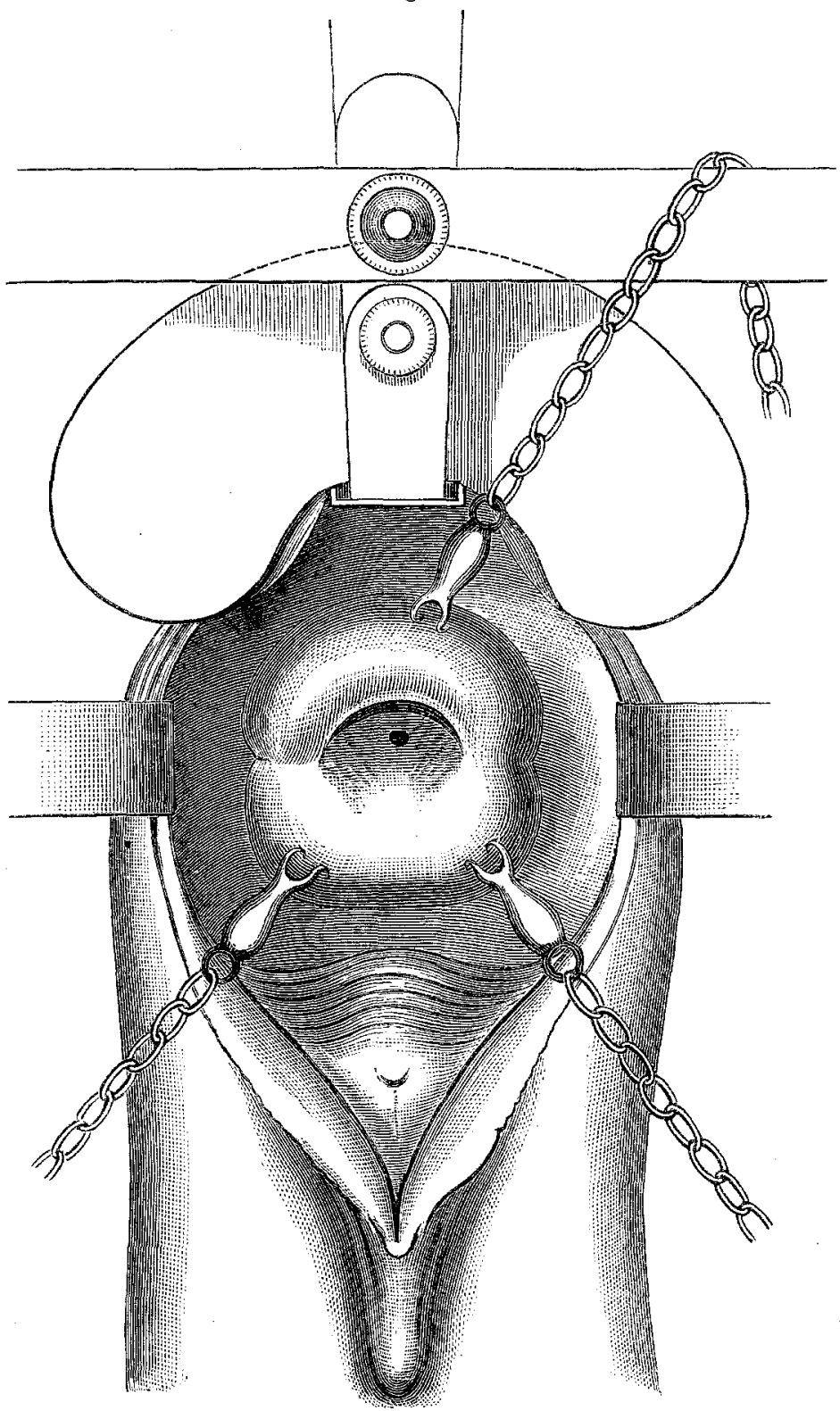

Fistula vesico-uterina (2 mm). Mereda Awrach, 1872.

operativer Versuch des unmittelbaren Nahtverschlusses am 21. November und am 30. December erfolglos. Endlich Metrokleisis (10 Nähte in die 
tief trichterförmige Wunde) am 23. Januar 1873. Nähte am siebenten Tage entfernt. Erste Vereinigung. Die Regel trat alsbald nach der Operation vorgreifend ein. Am 7. Februar 1873 geheilt von der Incontinenz entlassen. (Fig. 2 und 3.) (L. A. Neugebauer; L.-V. Nr. 53.)

Fig. 2.

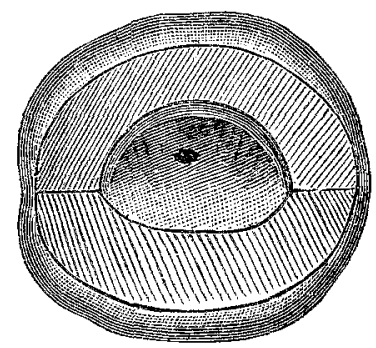

Fig. 3.

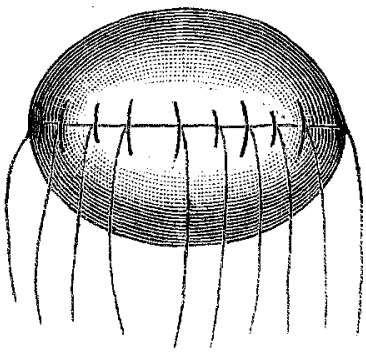

Mereda Awrach. Hysterokleisis cruenta. Anfrischung und Naht.

4. (Fall $124 \mathrm{der}$ Gesammtcasuistik): Fistula utero-cervicovesicalis. Syringokleisis immediata, sanatio incompleta, metrokleisis cruenta (mit 2 Nachoperationen), sanatio completa. - Keila Kleinmann, jüdische Landmannsfrau aus Swiniar (Gouvernement Radom), 30jährige V para, trat ein am 4. August 1865. In elfjähriger Whe vier lebende Kinder leicht geboren, das fünfte todt. Am 30. April 1865 Wasserabfluss bei Querlage, Vorfall einer Hand am 2. Mai mit Bewegung der Finger; am 3. Mai machten sich einige Frauen aus der Nachbarschaft iber die Gebärende her, zogen zunächst an dem vorgefallenen Arme, zogen den Rumpf und die Beine, sodann den innen bleibenden rechten Arm, endlich den Kopf heraus (nach Art der Selbstentwickelung). Sofort Harnfluss durch die Scheide mit gänzlicher Incontinenz, fünf Wochen schwer krank gelegen. - Zweimal Regel bis August. Beim Eintritte in die Klinilk nach $3 \frac{1}{2}$ Monaten: $4 \mathrm{~mm}$ weite Blasengebärmutterfistel entdeckt, Muttermund klaffend, stark zerrissen; Dank dem natürlichen Ektropium der nur theilwejse exhaltenen Muttermundslippen liess sich die Fistel einigermaassen einstellen. Am 5. August unmittelbarer Nahtverschluss mit fünf Silberdrahtnähten; Erfolg unvollkommen, daher am 27. October Metrokleisis mit Verschluss in longitudineller Richtung (Nähte von rechts nach links. Zwei kleine Nachoperationen. Vollkommen geheilt entlassen am 5. Februar 1866. Einige Tage nach der ersten Nachoperation Periode durch Scheide and Harnröhre zugleich).

(Diese Beobachtung sollte chronologisch als die zweite unserer Casuistik verzeichnet sein, aus Rücksicht aber auf die bereits im ersten Theile dieser Arbeit gedruckten Nummern der drei Fälle 4:3, 44 und 52 musste ich dieselbe hier einfïgen.) 
Fig. 4.

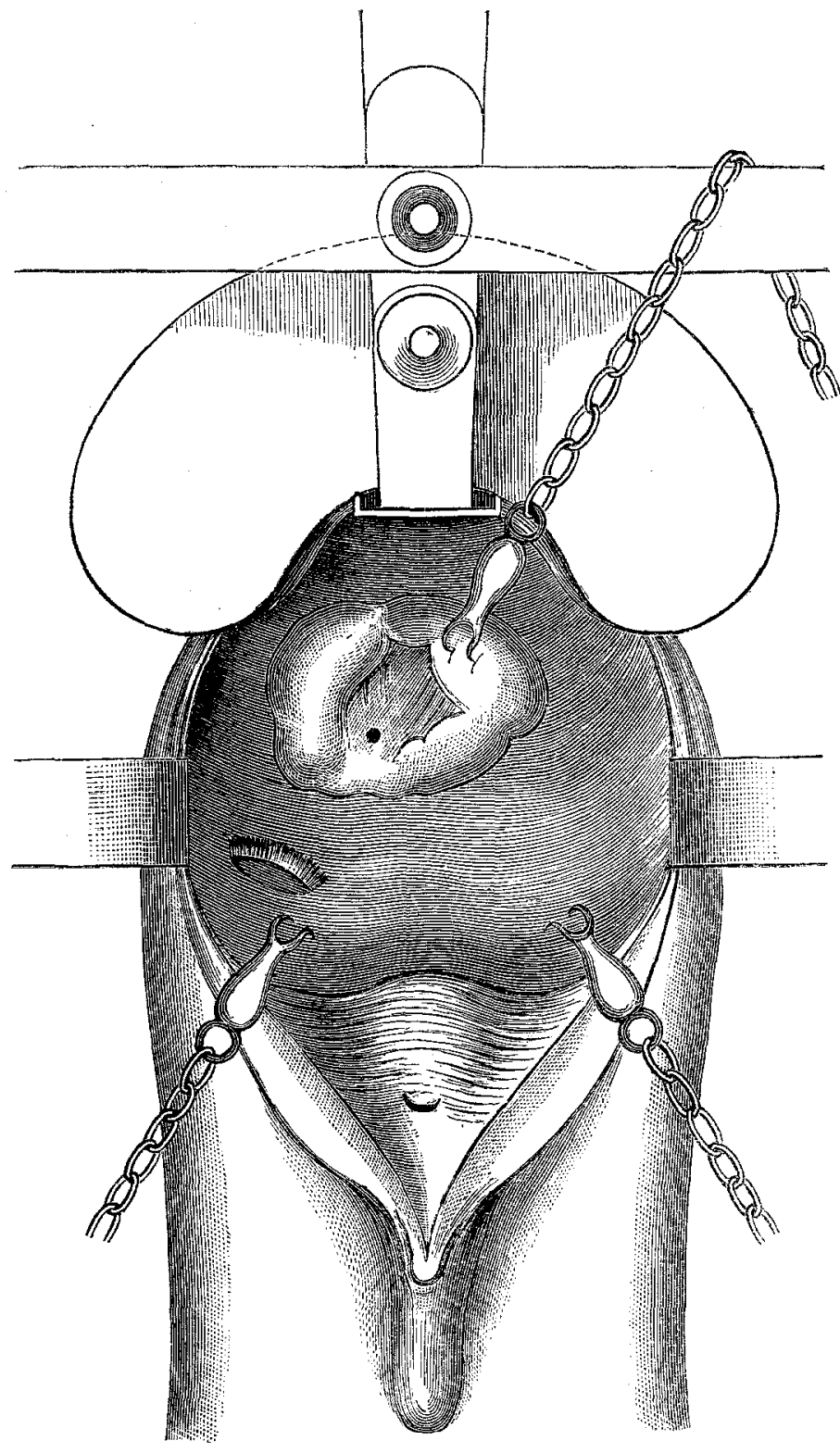

Fistula urogenitalis duplex (vesico-uterina, $1 \mathrm{~mm}$ lata, vesico-vaginalis, $13 \mathrm{~mm}$ lata), portio vaginalis uteri destrueta, atresia canalis cervicalis uteri. (Ryfka Rathmann, 1877 [siehe S. 6].) 
150 Neugabauer, 97 Beobachtangen vou Vesica-Cervico-Uterinfisteln。

5. (Fall 125 der Gesammtcasuistik): Fistula vesicalis colli uteri: Syringokleisis immediata. Sanatio. - Marjanna Godlewska, 31 jährige II para, trat ein am 30. October 1876: Zwei Zangenoperationen, zuletzt vor zwei Jahren nach dreitägiger Geburtsarbeit. $2 \mathrm{~mm}$ weite Fistel oberhalb des Muttermundssaumes. Unmittelbare Anfrischung und Naht (elf Nähte) am 2. November 1876, am 9. November Nähte entfernt, am 15. vollkommen geheilt entlassen. (In russischex Sprache veröffentlicht 1877 ; s. L. V.: L. A. Neugebauer.)

6. (Fall 126 der Gesammtcasuistik): Fistula vesico-vaginalisetfistulavesico-cervico-uterina una cumatresiacanalis cervicalis uteri supra fistulam. Syringokleisisimmediata fistularum. Sanatio. - Ryfka Rathmann, rhachitische

Fig. 5.

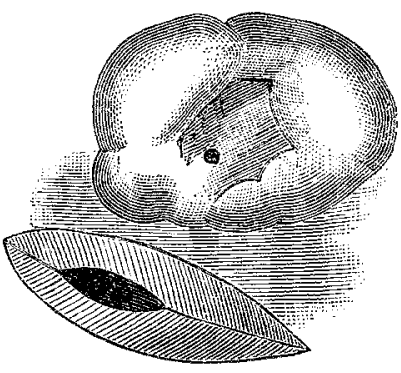

Fig. 7.

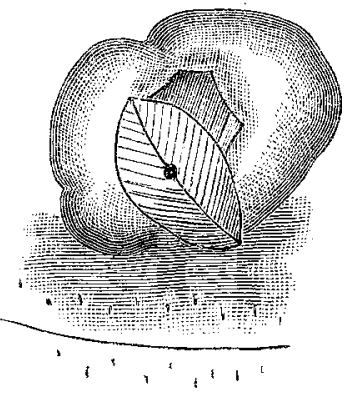

Fig. 6.

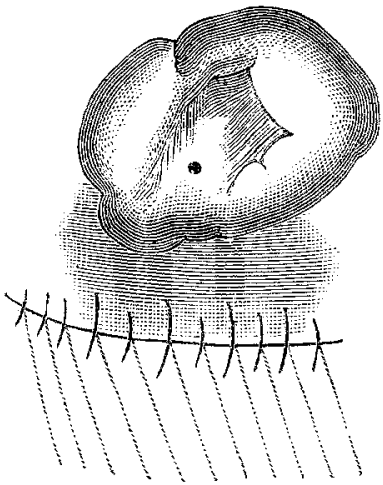

Fig. 8 .

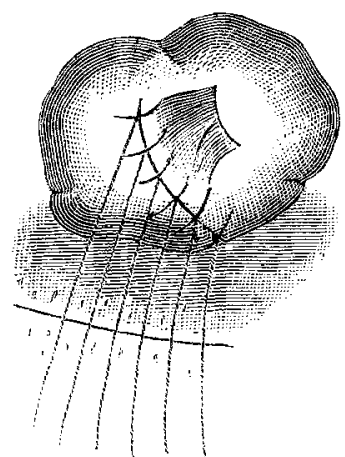

jüdische Händlersfrau, 26 jährige I para aus Skil, trat ein am 3. Juli 1877, nachdem sie am 18. April 1877 eine schwere Entbindung von fünftägiger Dauer durchgemacht batte. Natürliche Geburt eines todt- 
faulen Kindes in Schädellage. Harnfluss durch die Scheide vom achten Tage an. Elliptische linkseitig gelegene Blasenscheidenfiste 1 von $9 \mathrm{~mm}$ Längendurchmesser, $13 \mathrm{~mm}$ unterhalb der Muttermundslippe und - eine $2 \mathrm{~mm}$ breite Cervicalfistel im supravaginalen Theile des Mutterhalses. Vordere Lippe zerstört, Uterus nicht vergrössert, Periode noch nicht wieder erschienen. (Siehe Fig. 4-8.) Am 4. Juli 1877 blutiger Verschluss der Blasenscheidenfistel mit elf Kupferdrahtnähten in Gegenwart der Herren Drr. Hensel, Kondratowicz, Jasiński. Am 11. Juli Nähte entfernt, erste Vereinigung. Am 31. Juli Cervicalfistel unmittelbar geschlossen nach Ablösung der Schleimhaut um die Fistel herum in Gestalt eines anverletzten Ringes im Beisein der Herren Drr. Kwaśniewski, Hensel, Gerlach. Sechs Kupferdrahtnähte in schräger Richtung von oben links nach unten rechts. Verweilkatheter. Am 7. August Nähte entfernt. Erste Vereinigung. Geheilt entlassen am 16. August 1877.

7. (Fall 127 der Gesammtcasuistik): Harnblasen-Gebärmut terhalsfistel durch unmittelbaren Nahtverschluss mit

Fig. 9.

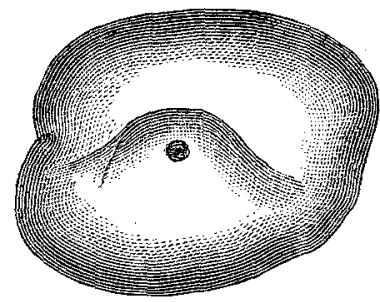

Hanna Pila, 1877.
Fig. 10.

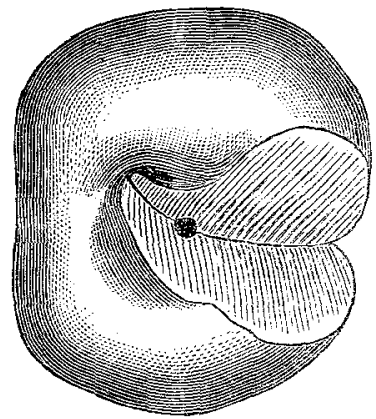

einmaliger Operation geheilt. (Nr. 158 des Fisteljournals der Klinik.) - Hanna Pila, 28jährige jüdische Krämersfrau aus Starezew, Ipara, klein von Wuchs, trat ein am 31. October 1877. Am 24. Mai verschleppte Entbindung in Stirnlage, natürliche Austreibung eines todten Kindes 60 Stunden nach Wasserabfluss. Nach sieben Tagen Harnträufeln (siehe Fig. 9, 10, 11). Rundliche $2 \mathrm{~mm}$ breite Cervicalfistel in der Mittellinie, $7 \mathrm{~mm}$ oberhalb des Muttermundssaumes. Linksseitiger Cervixriss. Regel schon mehrmals nach der Entbindung erschienen. An 6. November 1877 : nach rechtsseitiger Fig. 11. Cervixspaltung Anfrisehnng so, dass die Fistel in die neugeschaffene Cervixwunde miteinbegriffen wird. Schräge Nahtflucht von links oben 
nach rechts unten, sechs Nähte. Verweilkatheter nicht vertragen, nach 24 Stunden entfernt. Am 13. November Nähte entfernt. Erste Vereinigung. Am 2. December 1877 geheilt entlassen. Operation in Gegenwart des klinischen Auditorium

8. (Fall 128 der Gesammteasuistik): Fistula vesico-uterina simul cum fistula uretero-vesico-vaginali. Syringokleisis immediata (je eine Operation). Sanatio. - Antonie Wiśniewska, 30jührige Tagelöhnersfrau aus Wymysłow, IV para, trat ein am 25. Januar 1878. Drei leichte Eutbindungen, vierte schwer (Zange 20 Stunden nach Wasserabfuss, todtes Kind), sofort Harnfluss durch die Scheide, $4 \mathrm{~mm}$ weite Blasengebärmutterfistel $1 \mathrm{~cm}$ oberhalb des Muttermundssaumes in der Mittellinie zugleich mit einer sehr grossen mit linksseitiger Harnleiterfistel und Hervorstülpung der Harnblase durch đie Fistelöffnung verwickelten Blasenscheidenfistel. Die Harnleiterfistel sass in der linken Ecke des oberen Randes der Blasenscheidenfistel, die Oeffnung liess einen Sondenknopf durchgehen und fithrte in den unterhalb dieser Stelle zerstörten Harnleiter. Am 20. Februar 1878 in Gegenwart des Herm Dr. Michels Operation der Blasenscheidenfistel. Erste Vereinigung. Am 19. März Nahtverschluss der Cervicalfistel: beiderseitige Discisio colli bis etwas oberhalb des Niveau der Fisteloffnung, diesmal tief trichterförmige Anfrischung; die Wunde stellt sich nachenförmig querverlaufend dar. Fünf Nähte schliessen die Fistel und je eine $\mathrm{Naht}$ die beiden Cervixschnitte. Am 26. März Nähte entfernt, erste Vereinigung, geheilt entlassen am 2. April 1878.

9. (Fall 129 der Gesammtcasuistik): Fistula vesico-uterina. Incontinentia lotiisublata consutione cruenta oris uteri. Sanatio (Fig. 12, 13, 14). - Johanna Zajdowska, 35jährige

Fig. 12.

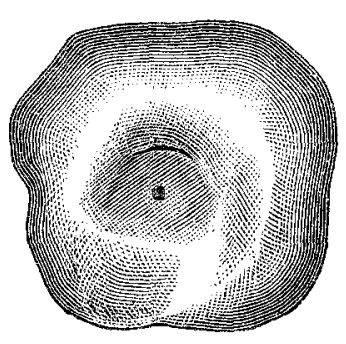

Fistula vesico-uterina. Hysterokleisis cruenta. Johanna:Zajdowska, 1878.
Fig. 13.

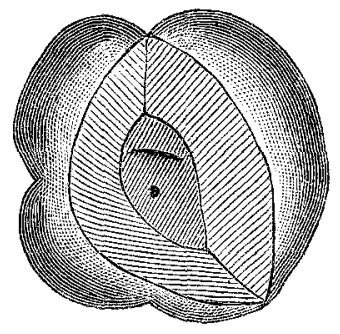

Fig. 14.

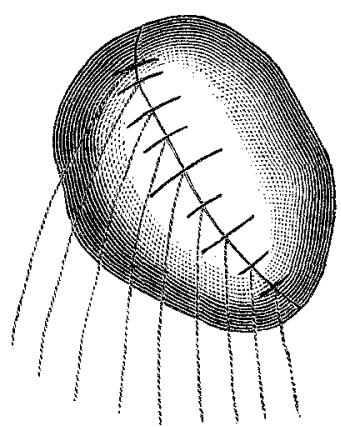

VII para, Bauersfrau aus Galki, trat ein am 2. Januar 1878. Sechs leichte Geburten lebender Kinder, letzte (siebente) Entbindung schwer, verschleppt, künstlich durch Perforation von der Hebamme beendigt bei 
Stirnlage, worauf der Umstand hinzuweisen scheint, dass ein Auge aus der Orbita herausgepresst war. Sofort'nach Extraction des Kindes Harnfluss durch die Scheide. Erst nach sechs Monaten lernte die Kranke zeitweilig etwas Harn zurïckzuhalten, ein 'Theil floss jedoch beständig ab. $2 \mathrm{~mm}$ breite Cervicalfistel $1 \mathrm{~cm}$ oberhalb des Muttermundssaumes. Am 16. Januar Periode. Muttermundslippen theilweise zerstört, Narbenschrumpfung, brüchiges Gewebe und schwerer Zutritt zur Fistel, somit am 7. Februar 1878: Hysterokleisis in Assistenz des Herrn Dr. Kondratowicz. Schrägliegende kahnförmige Wunde, neun Golddrähte, Verweilkatheter. Am dritten Tage Katheter, am siebenten Tage Nähte entfernt. Erste Vereivigung. Geheilt von der Incontinenz am 28. Februar 1878 entlassen.

10. (Fall 130 der Gesammtcasuistik): Fistula vesico-uterina pulsilla syringokleisi immediata sanata (einmalige Operation). - A n tonie Banas zukiewi c z, 24 jährige I para, Nähterin aus Radośzyce, trat ein am 6. Mai 1878. Am 26. December 1877 sechs Wochen zu früh durch schwere fünftägige Geburt von einem todten Kinde entbunden. Plattes Becken mit Conjugata vera des Einganges von $8 \mathrm{~cm}$. Vom achten Tage an Harnfluss, $1 \mathrm{~mm}$ breite Cervicalfistel in der Mittellinie oberhalb der zerstörten Vorderlippe. Am 28. Mai Operation: sechs Kupferdrähte durch die etwa $2 \mathrm{~cm}$ lange, schräg vom rechten Muttermundswinkel nach links unten gerichtete Wunde. Nach Entfernung der Nähte am siebenten Tage erste Vereinigung nachgewiesen. Aus Vorsicht noch für drei Tage der Verweilkatheter belassen. Am 26. Juni geheilt entlassen.

11. (Fall 131 der Gesammtcasuistik): Fistula vesico-cervicouterina post dilatationem vaginae cicatricibuscoarctatae sanata cauterisatione ope argenti nitrici (Nr. 179 des klinischen Fisteljournals). - Josephine Szturębska, 44jährige VII para, Tagelöhnersfrau aus Jeziorno, trat am 28. April 1880 ein. Im Juli 1879 ihre letzte Entbindung: 24 Stunden nach Wasserabfluss Zange, todtes Kind. Nach einer Woche schon Harnfluss durch die Scheide. Portio vaginalis uteri zerstört, etwas oberhalb des Scheidenansatzes in der vorderen Cervicalwand eine feine, $1 \mathrm{~mm}$ breite Cervicalfistel. Binnen drei Wochen siebenmal scheinbar erfolglos mit Höllenstein geätzt, endlich Nahtverschluss beschlossen. Da aber die Scheide durch Narben sehr verengt war, so erst Narbenincisionen und Dilatation der Scheide am 6. Juni. Nach drei Tagen wieder betrachtet. Die Kranke blieb merkwürdigerweise jetzt trocken und es stellte sich heraus, dass die Fistel fast ganz geschlossen war. Es wurde daher noch einmal geätzt (achtmal im Ganzen). Nach vier Tagen Verschluss vollständig. Am 21. Juni geheilt entlassen.

12. (Fall 132 der Gesammtcasuistik): Fistula vesico-uterina syringokleisi immediata ocelusa (Heilung durch einmalige Operation) (Nr. 182 des klinischen Fisteljournals). - Magdalene Rokita', ältere Bauersfrau aus Wiktoryn, trat am 12. Mai 1880 wegen Incontinenz seit ihrer am 11. December 1879 erledigten 


\section{Neugebauer, 27 Beobachtungen ron Vesico-Cervico-Uterinfisteln.}

neunten Entbindung ein: Natirliche Austreibung eines todten Kindes nach viertägiger Geburtsarbeit. Harnfluss vom zwölften Tage ab. $2 \mathrm{~mm}$ breite Cervicalfistel rechterseits oberhalb der Scheideninsertion. Portio vaginalis uteri zerstört, Incontinenz vollständig. Am 20. Mai Operation. $2 \mathrm{~cm}$ lange kahnförmige Wunde, sechs Nähte in sagittaler Richtung, am 27. Mai entfernt, erste Vereinigung. Am 9. Juni 1880 geheilt entlassen.

13. (Fall 133 der Gesammtcasuistik): Fis tula vesico-uterina vasta, consutio immediata, sanatio incompleta; Cauterisatio primum argento nitrico, tum eauterio Paquelini. Metrophlebitis. Pneumonia. Mors 47. die post operationem. - Veronika Gruszezak, 40jährige VIII para, Tagelöhnersfrau von mittlerer Körpergrösse, hat acht Mal rechtzeitig geboren, stets ohne Kunsthitlfe, Kopf voran. Die 1., 2., 4., 5. u. 7. Entbindung leicht, die 3., 6. und 8. schwer, besonder's aber die letzte (im März 1880), die erst drei Tage nach Wasserabfluss beendigt war. Harnfluss vom dritten Tage nach der Entbindung an. Trat in das Spital ein am 1. December 1881, zwei Wochen nach der letzten Menstruation. Querliegende, elliptische 7 und $4 \mathrm{~mm}$ in den Durchmessern haltende Cervicalfistel. Am 23. December 1881 unmittelbarer Fistelverschluss mit sieben Drähten nach trichterförmiger Anfrischung. Am neunten Tage Nähte entfernt, Fistel erweist sich nicht ganz geschlossen. Am 10. Januar 1882 Höllensteinätzung. Am 18. Januar Periode. Am 24. Januar Cauterisation mit Paquelin's Glühapparat. Der Harnfluss vermindert sich, aber es stellte sich Fieber ein, Koliken, Lendenschmerzen. Am 30. Januar Anzeichen von Pneumonie, erst rechts, dann auch links. Trotz zeitwelliger Besserung traten Durchfälle hinzu und die Kranke erlag am 8. Februar 1882 einer Metrophlebitis und metastatischen Pneumonie im Anschlusse an die Cauterisation mit Paquelin's Brennapparate. Bei der Leichenschau Eiterherde gefunden im linken Parametrium längs einiger thrombosirter Venen.

14. (Fall 134 der Gesammtcasuistik): B lasengebärmutterhalsfistel, Heilung durch unmittelbaren Nahtversehlus mit zweimaliger Operation. - Salomea Szymanik, 46 jährige IX para, trat ein am 1. December 1881. Die ersten acht Entbindungen waren leichte gewesen, stets lebende Kinder. Die neunte Entbindung (am 1. October 1881) verschleppt, schwer, durch Excerebration zwei Tage nach Wasserabfluss beendigt. Vom sechsten Tage nach der Entbindung an Harnfluss durch die Scheide. $2 \mathrm{~mm}$ breite Cervicalfistel oberhalb der zerstörten vorderen Lippe. Vom 3. bis zum 6. December Periode. Am 21. December Nahtverschluss, ohne Katheter belassen. Am 29. December Nähte entfernt. Unvollständige Heilung. Am 20. Januar 1882 Paquelin-Aetzung. Am 28. Januar Operation wiederholt: 10 Nähte, am 4. Februar entfernt, erste Vereinigung. Am 17. Februar 1882 geheilt entlassen.

15. (Fall 135 der Gesammtcasuistik): Fistula vesico-uterina, metrokleisis, sanatio. - Franziska Pośnik, 45jährige XIIp., 
Banersfran aus Kamien, von hohem Wuchs, trat ein am 2. October 1882. Alle Entbindungen ohne Kunsthülfe, die 7., 9. u. 12. schwer: todte Kinder. Letzte Entbindung am 20. October 1881: 24. Stunden nach Wasserabfluss beendigt, fünf Tage darauf Harnfluss durch die Scheide. $2 \mathrm{~mm}$ breite Cervicalfistel $13 \mathrm{~mm}$ oberhalb des Muttermundssaumes. Muttermund klafft weit infolge von Cervixeinrissen. Fistel hinter einer narbigen Falte verborgen und sehr schwer zugänglich. Da ein unmittelbarer Nahtverschluss kaum ausfübrbar erschien, zunächst Versuche mit Höllensteinätzung und zwar 18 Cauterisationen im Verlaufe von acht Wochen - erfolglos. Zwei Mal Regeln während dieser Zeit. Am 13. December 1882 Metrokleisis cruenta unter Assistenz des Herrn Prof. Kosiński und der Studirenden. $1 \mathrm{~cm}$ breiter Anfrischungskreis, 11 Nähte. Bei Entfernung derselben am 22. December erste Vereinigung festgestellt. Bald darauf Menstruation durch die Harnröhre. Von der Incontinenz geheilt entlassen am 4. Januar 1883.

16. (Fall 126 der Gesammtcasuistik): $F$ istula vesicalis colli uteri simul cum fistula vesico-vaginali. Sanatio spontanea fistulae cervicalis ope cauterisationis argento nitrico, syringokleisis cruenta fistulae vesico-vaginalis. Sanatio. - Anna Biernacik, 27 jährige III para, Kolonistenfrau aus der Gegend von Rawa, trat ein am 6. Mai 1882: nur das erste and dritte Kind lebend geboren, das zweite todt. Letzte Entbindung am 5. April 1882: Forceps (Dr. Krupski), 40 Stunden nach Wasserabfluss; vom dritten Tage an Harnfluss. Nach viermaliger Höllensteinätzung erwies sich bei Milchprobe am 25. Mai die Cervicalfistel verheilt. Am 10. Juni Blasenscheidenfistel vernäht in Gegenwart des Dr. Kapliński und stud. Widera; bei Entfernung der Nähte am 17. Juni erste Vereinigung, am 27. Juni geheilt entlassen.

17. (Fall 137 der Gesammtcasuistik): Blasengebärmutterhalsfistel. Heilung dureh unmittelbaren Nahtverschluss mit ein maliger Operation (Fig. 15, 16, 17). - Natalie Olesz-

Fig. 15.

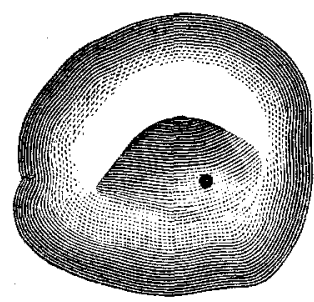

Fig. 16.

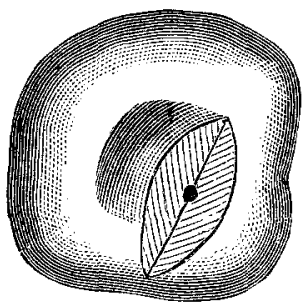

Natalie Oleszkiewicz, 1883.

Fig. 17.

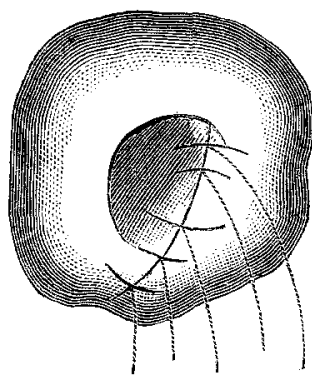

kiewicz, 22 jährige II para, Conditorsfrau von kleinem Wuchs und zartem Körperbau, gut genährt, trat am 21. März 1883 wegen Incontinenz ein. Zwei todte Kinder geboren am 6. Februar 1881 und 
$156^{\circ}$ Neugebauer, 27 Beobachtungen von Vesico-Cervico-Uterinfisteln.

am 3. Februar 1883. Erste Entbindung endigte ohne Kunsthủlfe bei Gesichtslage fünf Tage nach Wasserabfluss, die zweite - ebenfalls bei Gesichtslage - wurde mittels der Zange beendigt (Dr. Tyrchowski jun.). Vom siebenten Tage nach der Entbindung an Harnfluss durch die Scheide. Kleine Cervicalfistel $13 \mathrm{~mm}$ oberhalb des Muttermundssaumes, rechterseits. Am 22. März 1883 Periode. Am 5. April Operation im Beisein der Herren Dr. Kaplinski und Rogoziński und stud. med. Ciagliński. Fünf Nähte, schrägg liegende Nahtlinie. Am 11. April bei Entfernung der Nähte erste Vereinigung. Am 22. April geheilt entlassen.

18. (Fall 138 der Gesammtcasuistik): Fistula vesico-uterina. Heilung durch blutigen Muttermundsverschluss, zweimalige Operation. - Marianne Bańkowska, 36 jährige VIIp., trat ein am 22. Februar 1883. Letzte Entbindung im Frühjahre 1882. Stets ohne Kunsthülfe entbunden; einmal Zwillinge. Von den acht Kinder'a sind vier todt geboren (von den Zwillingen der erste lebend, der zweite todt). Die Zwillingsgeburt (die funfte Entbindung) war die schwerste und dauerte volle vier Tage. Seit jener fünften Entbindung bestand eine Blasencervixfistel. Die Incontinenz war sofort bei der Austreibung des zweiten Zwillinges entstanden. Runde, $1 \mathrm{~mm}$ breite Fistel im untersten Theile der Cervicalwand. Uterus wenig beweglich. Keine Periode seit der letzten Entbindung. Viermalige Aetzung der Fistel mit Höllenstein blieb erfolglos, Am 23. März 1883 Metrokleisis cruenta, sehr schwierig wegen Unbeweglichkeit des Uterus. Neun Messingdrähte, unvollkommene Heilung. Am 11. Mai Wiederholung der Operation: acht Drahthefte, am 18. Mai entfernt. Erste Vereinigung. Am 20. Mai 1883 geheilt entlassen.

19. (Fall 139 der Gesammtcasuistik): Fistula vesico-uterina. Sanatio spontanea. - Rosalie Kozera, 42 jährige IX para, Bauersfrau, trat am 29. September 1883 ein. Das 1. nnd 3. bis 6. Kind lebend, das 2,, 7., 8. und 9. Kind todtgeboren; letzte Entbindung im Juli 1883 dauerte bis 58 Stunden nach Wasserabfluss. Vom 9. Tage an Harnfluss durch die Scheide. Cervix beiderseits stark eingerissen, $1 \mathrm{~mm}$ breite Vesicouterinfistel, $13 \mathrm{~mm}$ oberhalb des Muttermundssaumes. Da die Kranke beständig fieberte und sehr heruntergekommen erschien, wurde vorläufig von der Operation Abstand genommen und zunächst eine vorbereitende Allgemeinbehandlung eingeleitet und sollte die Kranke nach zwei Monaten sich wieder melden; sie kam jedoch nicht wieder in das Spital zurück, da die Fistel mittlerweile von selbst geheilt war.

20. (Fall 140 der Gesammtcasuistik): Fistula vesico-uterina cervicalis, Syringokleisis immediata. Sanatio incompleta. - Lucie Sa la, ?jährige II para, Tagelöhnersfrau aus Gromadzice; erste Entbindung leicht, zweite schwer: Wendung 77 Stunden nach Wasserabfluss, todtes Kind, sofort Harnfluss, vollkommene Incontinenz. Trat ein am 23. März 1884: $2 \mathrm{~mm}$ weite Cervicalfistel oberhalb des Saumes der zerstörten Vorderlippe. Die Umgebung der 
Fistel noch in Eiterung begriffen. Kamillentheespülungen und Aetzung der Granulation, um die Uebernarbung zu erzielen. Am 18. Mai unmittelbarer Nahtverschluss mit myrthenblattförmiger Anfrischung, Wunde durch acht Nähte verschlossen. Nahtlinie schräg von links oben nach rechts unten. Unvollkommene Heilung. Die Kranke verlor noch theilweise Harn dureh die Scheide nach Entfernen der Nähte, musste jedoch infolge Schliessung der Klinik wegen der beginnenden Sommerferien am 6. Juni vorläufig entlassen werden. Da sie sich nicht wieder gemeldet hat, dürfte man vermuthen, die nachgebliebene Fistel sei von selbst nachträglich zum Verschluss gelangt (?).

21. (Fall 141 der Gesammtcasuistik): Blasengebärmutterhalsfistel durch unmittelbaren Nahtverschluss mit einmaliger Operation geheilt (Nr. 253 des klin. Fisteljournals). Franziska Jakubik, 25jährige I para, Bauersfrau aus Siedlce, trat am 16. Januar 1885 ein. Am 14. September 1884 rechtzeitig und natürlich entbunden, 80 Stunden nach Wasserabfluss. Schädellage, todtes Kind. Vom siebenten Tage an Harnabfluss dureh die Scheide. Gegenwärtig Incontinenz nur zeitweise. $2 \mathrm{~mm}$ breite Cervicalfistel in der Mittellinie oberhalb der zerstörten vorderen Lippe, die hintere Lippe ist in drei Theile gespalten. Am 13. Februar Operation: schräg liegende Anfrischungswunde von $2 \mathrm{~cm}$ Länge und $7 \mathrm{~mm}$ Breite. Fünf Nähte am 20. Februar entfernt, erste Vereinigung. Am 7. März geheilt entlassen.

22. (Fall 142 der Gesammtcasuistik): Fistula vesic 0 -uterina, incontinentia lotii hysterokleisi sublata. - Johanne Górnicka, 20 jährige I para, ledig, trat am 17. November 1886 ein. Im Februar 1884 entbunden. Querlage mit vorgefallenem Arm, die Hebamme schnitt denselben $a b$, holte alsdann den zweiten Arm herab, schnitt auch diesen aus und machte dann die Wendung und Extraction - 37 Stunden nach Wasserabfluss. Sofort Harnfluss durch die Scheide. Grosse $8 \mathrm{~mm}$ breite Cervicalfistel (instrumentellen Ursprunges?) im supravaginalen Theile der Cervix. Vordere Iippe gänzlich zerstört, Fistelränder ungemein dünn und scharfrandig. Am 10. November HysterokJeisis mit acht Nähten, die am 18. November entfernt wurden. Erste Vereinigung. Von der Incontinenz geheilt entlassen am 3. December 1886.

23. (Fall 143 der Gesammtcasuistik): Blas engebärmutterhalsfistel - Operation zeitweilig a ufgeschoben wegen Nephritis. - Antonie Lewandowska, 34jährige Ipara, trat am 30. November 1886 ein wegen Incontinenz seit ihrer Entbindung vor zwei Jahren. Wegen schlechten Allgemeinbefindens, Decrepidität und chronischer Nephritis Operation vorläufig anfgeschoben.

24. (Fall 144 der Gesammteasuistik): Fistula vesico-uterina, incontinentia lotii hysterokleisi cruenta sublata. Wiederholte Operationen. - Marianne Wróbel, 38jährige III para, Popenfrau, trat ein am 17. September 1886. Menses vom 15. Jahre an, 
hat drei Mal geboren, vor neun und vor fünf Jahren und vor vierzehn Monaten (11. Juni 1886). Die ersten beiden Kinder lebend, das letzte todt geboren. Stets natiuliche, aber stets verschleppte Entbindung, bis 48 Stunden nach Wasserabfluss. Zehn Tage nach der letzten Entbindung begann Harnfluss durch die Scheide. Vollständige Incontinenz bis jetzt. Grosse $1 \mathrm{~cm}$ breite Fistel $1 \mathrm{~cm}$ oberhalb des Muttermundssaumes. Wegen der Grösse der Fistel, Unnachgiebigkeit ihrer Ränder und narbiger Beschaffenheit Verzicht auf unmittelbaren Verschluss, also Metrokleisis, aber auch diese Operation nicht auf einmal in voller Ausdehnung wegen starker Zerklüftung der Muttermundslippen. Am 12. October 1886 wird die rechte Hälfte und der mittlere Theil des Muttermundes verschlossen, es blieben jedoch nach Entfernung der Nähte drei kleine Oefinungen, somit einschliesslich des linken Muttermundswinkels vier Oeffnungen zu schliessen. Leichte Parametritis, die jedoch schnell zurückging. Am 17. November zeitweilig entlassen, kommt die Kranke am 6. Mai 1887 wieder. Am 11. Mai Vernähung der erwähnten drei kleinen Nachfistelchen mit sieben Nähten, aber nur eine davon erwies sich beim Entfernen der Nähte verheilt. Am 25. Mai 1887 Vernähung des linken noch nicht operirten Mattermundswinkels (durch den Assistenten). Am 20. Juni endlich noch die zwei kleinen, von der ersten Operation nachgebliebenen Fistelchen angefrischt und vernäht mit breiter elliptischer Anfrischung. Beim Entfernen der Nähte erwies sich nun der Muttermund völlig geschlossen und wurde somit die Kranke geheilt entlassen.

25. (Fall 145 der Gesammtcasuistik): Fistula vesico-uterina syringokleisi immediata sanata (einmalige Operation). Franziska Kleszcz, 45 jährige IV para, Kaufmannsfrau aus Czemierniki, trat am 25. April 1887 ein. Vier rechtzeitige Entbindungen ohne Kunsthülfe, zuletzt vor acht Wochen. Das tief asphyktische Kind stirbt bald. Dauer der letzten Entbindung vom Wasserabflusse an 48 Stunden. Nur die letzte Entbindung war schwer, der Harn Hoss sofort durch die Scheide ab. Lippen theilweise zerstört, $2 \mathrm{~mm}$ breite Cervicalfistel. Am 4. Mai 1887 unmittelbarer Nahtverschluss mit breiter elliptischer Anfrischung. Sechs Nähte. Verweilkatheter. Am 10. Mai Nähte entfernt, erste Vereinigung; am 19. Mai 1887 geheilt entlassen.

26. (Fall 146 der Gesammtcasuistik): Fistulavesico-uterina - Cauterisatio ope argenti nitrici. - Marianne Gajda, 40jährige IX para, Bauersfrau von hohem Wuchs, $153 \mathrm{~cm}$, mit plattem nichtrhachitischem Becken (Conjugata vera 7,5, Conjugata diagonalis 9,5, Conjugata externa $17,0 \mathrm{~cm}$; Symphysis pubis $5,5 \mathrm{~cm}$ hoch; Linea bitrochanterica $30,5 \mathrm{~cm}$, Linea bicristalis ossium ilei $25,0 \mathrm{~cm}$, bispinalis anterior superior 24,5 cm), tritt am 3. October 1888 wegen Harnincontinenz seit ihrer letzten Fntbindung vor sieben Monaten ein. Im 23. Lebensjahre verheirathet, hat neun Mal geboren und zwar alle Kinder in Schädellage, natïrlich aber schwer. Die dritte und vierte Schwangerschaft endeten mit einem Abort in der ersten Hälfte 
der Schwangerschaft, das fünfte Kind todtgeboren, das letzte starb am dritten Tage nach der Geburt, welche drei Tage gedauert hatte. Incontinenz vom achten Tage an, anfangs vollständig, jetzt zeitweise. Es handelt sich um eine fast stecknadelkopfgrosse Fistelöffnung im Cervicalkanale, 1,5 cm oberhalb des Saumes der stark zerrissenen Vorderlippe. Es gelingt nach Herabziehen der frei beweglichen Gebärmutter unter Herstellung eines künstlichen Ektropium der Muttermundslippen mittels gestielter Häkchen die Mündungsöffinung der Fistel im Gesichtsfelde einzustellen. Der Harn enthält noch immer Eiterflocken, die Kranke fiebert öfters und klagt über Schmerz in der linken Nierengegend. Vom 3. bis 10. October wurde fünf Mal mit Hö1lenstein geätzt. Die Kranke lag gewöhnlich nach der Aetzung einen Tag lang trocken, am übernäehsten Tage war sie wieder incontinent. Eine am 29. October ausgeführte Milehprobe ergab Fortbestand der Fistel.

27. (Fall 147 der Gesammtcasuistik): Fistula vesico-cervicouterina - Sanatio ope unius cauterisationis argento nitrico. - Klara Morozow (Widzicka), kräftig gebaute Bäuerin, 23 Jahre alt, war vom 19. bis 22. Jahre verheirathet, ist seit einigen Monaten Wittwe. Sie hat zwei Kinder geboren, das erste ohne Kunsthülfe rechtzeitig, lebend, das zweite todt vor jetzt drei Wochen. Der Arzt in der Stadt Bielsk entwickelte nach dreitägiger Dauer der Geburtsarbeit das Kind mittels der Zange. Vom siebenten Tage an Harnfluss durch die Scheide und zwar eine Woche lang vollständige Incontinenz, in der zweiten Woche nach der Entbindung wurde die Incontinenz zeitweise und kam die Kranke wegen ihres Harnflusses in die Klinik. Mässig plattes Becken mit ungewöhnlich hervorragendem Schambeinknorpel. Uterus noch ziemlich gross, schlecht zurüekgebildet, frei beweglich. Kein blutiger Ausfluss, Harn klar, wenig Harnekzeme. Querelliptische linsengrosse Cervicalfistel etwa $1 \mathrm{~cm}$ oberhalb des Saumes der mehrfach eingerissenen vorderen Muttermundslippe. Tiefer Cervicalriss rechts oben und links unten. Es gelingt nur sehr schwer die Fistel für das Auge einzustellen, obwohl der Cervicalkanal klafft und den Finger einlässt. Der Saum des äusseren Muttermundes ist ringsherum zackig. Es wird sofort eine Aetzung mit Höllensteinstift vorgenommen am Tage der Aufnahme am 15. October 1888, schon am 17. October hielt die Kranke bereits den grössten Theil des Urins zurïck und vom 18. October an blieb sie trocken. Nach 12 Tagen wurde eine Milchprobe gemacht. Die Cervicalfistel ist endgültig geschlossen. Wir waren Beide nicht wenig überrascht, eine verhältnissmässig grössere $(4-5 \mathrm{~mm}$ breit, wenn auseinandergezogen $2 \mathrm{~mm}$ hoch) Fistel so schnell heilen zu sehen unter dem Einflusse einer einzigen Aetzung. Ich glaube, dass die Rückbildung des puerperalen Uterus und die im Spital angeordnete ruhige Riickenlage im Bette ihren Theil haben bei dieser ïberraschend schnellen Heilung. Bei der letzten Besichtigung erschien der Saum der vorderen Muttermundslippe etwas eingerollt nach innen, hinter ihm linksseitig eine grubenförmige Vertiefung, bezw. trichter- 
160 Neugebauer, 27 Beobachtungen von Vesico-Cervico-Uterinfisteln.

förmige Einziehung; deren Grund dem Sitze dez ehemaligen Fistel entsprach. Geheilt entlassen Ende October 1888.

Fall 148: Die Beobachtung Dr. J. Rosenthal's betrifft eine jüdische Kranke (Rojza Kaufman a [Nr. $\left.\frac{384}{26} 1885\right]$ ) aus jüngster Keit in Warschau, welche jeden operativen Eingriff verweigerte nnd daher ungeheilt aus dem Spitale entlassen wurde.

(Fortsetzung folgt.) 